\title{
Factors Associated with First-Line Antiretroviral Therapy Failure amongst HIV-Infected African Patients: A Case-Control Study*
}

\author{
Charles M. Kwobah $^{1 \#}$, Ann W. Mwangi ${ }^{2,3}$, Julius K. Koech ${ }^{3}$, Gilbert N. Simiyu ${ }^{3}$, Abraham M. Siika ${ }^{1,4}$ \\ ${ }^{1}$ USAID-AMPATH, Moi University Clinical Research Centre, Eldoret, Kenya; ${ }^{2}$ Department of Behavioral Sciences, College of \\ Health Sciences, Moi University School of Medicine, Eldoret, Kenya; ${ }^{3}$ USAID-AMPATH Partnership, Biostatistics, Eldoret, Kenya; \\ ${ }^{4}$ Department of Medicine, College of Health Sciences, Moi University School of Medicine, Eldoret, Kenya. \\ Email: "ckwobah@yahoo.com
}

Received October $7^{\text {th }}, 2012$; revised November $5^{\text {th }}, 2012$; accepted November $12^{\text {th }}, 2012$

\begin{abstract}
Background: Since 2001, anti-retroviral therapy (ART) has been provided to over 75,000 HIV-infected patients at the USAID-Academic Model Providing Access to Healthcare (AMPATH) Partnership in western Kenya. Over 1000 of these patients have switched to second-line ART. We therefore set out to determine factors associated with first-line ART failure amongst these patients. Methods: This case controlled study matched patients (in the ratio 1:2) from the electronic AMPATH Medical Record System on the basis of age, gender, and ART initiation date. Cases were adults ( $\geq 18$ years) who initiated second-line ART between January 1, 2007 and July 31, 2011 after at least one viral load measurement $>5000$ copies $/ \mathrm{ml}$ or satisfying the WHO immunological or clinical failure criteria. Controls were those on non-failing first-line ART with a CD4 count $>400 / \mathrm{ml}$ within the last 12 months, at the time of case incidence. Conditional logistic regression for paired data was used to assess association. We evaluated the strength of association of risk factors using stratified Cox model. Results: Of the 1084 cases and 2149 controls included in the analysis, $62 \%$ were female. Median age was 36.5 years (IQR = 30.7 - 43.1); median baseline CD4 cell count was $161 / \mathrm{ml}(\mathrm{IQR}=72$ - 277); Median time to ART failure was 37 months (IQR $=24-47)$. Low baseline CD4 count $<50 / \mathrm{ml}(\mathrm{H} . \mathrm{R}=7.07,(95 \% \mathrm{CI}=$ 4.92 - 10.15); Zidovudine based ART (H.R 1.76, 95\% CI $=1.25-2.48$ ) and imperfect ART adherence (H.R = 2.77, $95 \% \mathrm{CI}=2.20-3.49)$ were independently associated with treatment failure. Conclusion: In this setting, low baseline CD4 count, zidovudine-based ART and imperfect adherence are associated with first-line ART treatment failure.
\end{abstract}

Keywords: HIV; Treatment; ART Failure

\section{Introduction}

Infection with the Human Immunodeficiency Virus (HIV) leads to depletion of the immune system and increases the risk of opportunistic conditions which are responsible for increased mortality [1-3]. Antiretroviral therapy (ART) restores immune function and reduces HIV-related morbidity and mortality $[4,5]$. This advantage is eroded when virological treatment failure develops. In the sub-Saharan Africa, many patients who experience virological failure do not switch to potent second line regimens due to resource limitation, yet those who remain on a failing first-line regimen experience disproportionately higher morbidity and mortality compared to those who switch

\footnotetext{
*Source of Funding: The AMPATH clinical care program is supported by the President's Emergency Plan for AIDS Relief (PEPFAR) through USAID under the terms of Cooperative Agreement No. 623-A-00-0800003-00. The authors do not report any conflict of interests.

\#Corresponding author.
}

[6,7]. Documented factors associated with virological treatment failure include poor adherence, certain ART regimen combinations, primary infection with drug resistant strains of HIV, prior exposure to antiretroviral monotherapy, high baseline plasma viral load and low baseline CD4 count [8-11].

Since 2001, ART has been provided to HIV-infected patients at USAID-Academic Model Providing Access to Healthcare (AMPATH) Partnership. AMPATH is a collaboration between Moi University School of Medicine (MUSoM), Moi Teaching and Referral Hospital (MTRH) and a consortium of North American Universities led by Indiana University. AMPATH works in partnership with the Kenya Ministry of Health to provide primary healthcare services including comprehensive HIV care to a catchment population of 4 million in western Kenya. As at the end of July 2011, AMPATH had enrolled over 130,000 HIV-infected patients, of whom over 75,000 had 
initiated ART. Over 1000 of these patients on ART had initiated second line therapy. We aimed to determine factors that contribute to patients failing their first-line ART in this large HIV cohort in sub-Saharan Africa, to provide the basis for interventions to mitigate them.

\section{Methods}

\subsection{Ethical Approval}

Ethical approval for retrospective analyses of routinely collected data was obtained from the Moi University/Moi Teaching and Referral Hospital Institutional Research and Ethics Committee and Indiana University School of Medicine Institutional Review Board. Patient informed consent was waived by the regulatory bodies.

\subsection{Setting}

This study was conducted at AMPATH, a large ambulatory HIV treatment program in western Kenya, with a HIV prevalence of $7.1 \%$ in the general population [12]. AMPATH has established 80 regional HIV treatment clinics in a predominantly rural community. Patient medical records are captured and stored in the AMPATH Medical Record System (AMRS), an electronic database which provided the sampling frame for this study [13]. The study included patients who were enrolled between January 1st, 2001 and July 31st, 2011.

The ART protocols in use during the time-frame of this study included 2 Nucleoside Reverse Transcriptase Inhibitors (NRTIs) plus 1 Non-Nucleoside Reverse Transcriptase Inhibitor (NNRTI) for first-line treatment. AMPATH patients therefore received combinations containing stavudine (D4T) or zidovudine (AZT) + lamivudine (3TC) + Nevirapine (NVP) or efavirenz (EFV). Tenofovir (TDF)-based regimens were introduced in 2010.

At enrolment, patients routinely have clinical, laboratory and radiological evaluations including physical examination, full blood count, alanine transaminase, serum creatinine, VDRL, CD4 count and chest X-ray. CD4 count is monitored six monthly. Viral load is only performed when treatment failure is suspected, based on clinical and immunological parameters.

ART was typically initiated when the CD4 count was $<250 / \mathrm{ml}$ or if the patient was in World Health Organization (WHO) clinical stage 3 or 4 regardless of the CD4 count. The cut-off CD4 count for initiating ART was revised to $350 / \mathrm{ml}$ in 2010 in line with the national and WHO ART guidelines. AMPATH ART guidelines also recommend routine universal Co-trimoxazole Prophylactic Therapy (CPT) and Isoniazid Prophylactic Therapy (IPT). Patients receive free comprehensive care including treatment for opportunistic infections (includeing tuberculosis), macro- and micro-nutrient supplemen- tation, psychosocial counseling, adherence counseling, prevention strategies and defaulter tracing. Adherence is assessed on every clinic visit by self report. Adherence is considered "perfect" when patients self report indicate that they have taken all doses at every visit since starting ART.

\subsection{Study Design and Data Sources}

We performed a matched case-control study. Data were extracted from the AMRS. Cases and controls were matched in the ratio of $1: 2$ based on age $( \pm 5$ years of case), gender and date of ART initiation ( \pm 30 days of case) using a SAS macro program created by Kosanke and Bergstralh that computes a distance matrix between a group of cases and a group of potential controls using variable optimal matching [14].

\subsection{Definition of Cases and Controls}

Cases were defined as adults ( $\geq 18$ years) who initiated second-line therapy between January 1, 2007 and July 31, 2011 after at least one viral load measurement $>5000$ copies/ml or satisfying the WHO 2006 immunological or clinical failure criteria [15]. Controls were those on nonfailing first-line ART with a CD4 count $>400 / \mathrm{ml}$ within the last 12 months, at the time of case incidence. Both cases and controls were required to have been on ART for at least six months. Second-line therapy was defined as a Protease-Inhibitor (PI) containing regimen after failure of an initial NNRTI regimen. All identified cases and controls were included in the analysis regardless of vital status at the time of the study. Controls were censored at their last clinic visit on first-line regimen.

A dataset was constructed containing the following categorical variables, determined a priori to be of significance in similar settings [8-11]: clinic type (rural versus urban), education (primary versus post-primary), baseline CD4 count $(<50 / \mathrm{ml}, 50-100 / \mathrm{ml}, 101-250 / \mathrm{ml},>250 / \mathrm{ml})$, baseline WHO clinical stage (stage 1, 2, 3 and 4), Body Mass Index at baseline $(<18.5, \geq 18.5)$, baseline hemoglobin level $(<10 \mathrm{~g} / \mathrm{dl}, \geq 10 \mathrm{~g} / \mathrm{dl})$, HIV disclosure status (Yes, No), travel time to clinic ( $<30 \mathrm{~min}, 30-60 \mathrm{~min}, 1$ $2 \mathrm{hrs},>2 \mathrm{hrs}$ ), HIV discordance at baseline (yes, no, unknown), NRTI backbone (Zidovudine, Stavudine), NNRTI anchor (Nevirapine, Efavirenz), ART perfect adherence (yes, no).

\subsection{Sample Size}

A total of 3233 participants (1084 cases and 2149 matched controls) were included in the analysis.

\subsection{Data Analysis}

All analyses were performed using Stata version 11. All 
tests of significance were two-sided with p-value less than 0.05 considered significant. Continuous variables were compared using the Wilcoxon rank-sum test; while categorical and dichotomous variables were compared using the chi-square test and Fishers exact test where appropriate. Conditional logistic regression for paired data was used to assess association. Survival analysis was performed for the primary outcome of time to first-line ART failure with survival time being defined as time from first-line ART regimen to second-line regimen switch.

\section{Matched Pair Analysis}

Multivariate analysis was performed by fitting a stratified Cox model on matched pairs to adjust for potential imbalance in the baseline and clinical characteristics for both cases and controls [16]. The ART exposure was analyzed in a "factorial" fashion where all participants who received Zidovudine were analyzed together, regardless of whether they received Efavirenz or Nevirapine. Likewise, all participants who received Nevirapine were analyzed together regardless of whether they received Stavudine or Zidovudine.

Variables used to match are by definition not included in the Cox model. Results were reported as the multivariate-adjusted odds ratios (AORs) with $95 \%$ confidence intervals (CIs) for the association between predictor variables and the outcome.

\section{Results}

Of the 3233 participants (1084 cases; 2149 controls) included in the final analysis: $62 \%$ were female; median age was $36.5(\mathrm{IQR}=30.7-43.1)$; median time on ART was 43.2 months $(\mathrm{IQR}=26.4$ - 57.6); median baseline CD4 cell count was $161 / \mathrm{ml}(\mathrm{IQR}=72-277)$. Median time to ART failure was 37 months (IQR $=24-47$ ). The commonest first-line ART regimens were: Stavudine/ Lamivudine/Nevirapine (d4t-3tc-nvp), 65.4\% $(\mathrm{n}=2114)$; zidovudine/Lamivudine/Nevirapine (zdv-3tc-nvp), $15.4 \%$ $(\mathrm{n}=497)$ and stavudine/Lamivudine/Efavirenz (d4t-3tcefv), $9.7 \%(n=314)$. Majority $(93.5 \%)$ of the cases had treatment failure confirmed virologically while the remainder were classified as immunological or clinical failures. Study subjects had a median CD4 count of 114.6 $(\mathrm{IQR}=61-182)$ and median viral load of $60,897(\mathrm{IQR}=$ $24,092-178,583)$ at the time of change to second line.

The overall mortality rate in this cohort was 7.7 per 1000 person years of follow-up $(95 \% \mathrm{CI}=6.25-9.519$; $\mathrm{n}=89)$ and was slightly higher among cases, $10.7(95 \%$ $\mathrm{CI}=7.6-15.1 ; \mathrm{n}=33)$ than controls $6.6(95 \% \mathrm{CI}=5.1$ $8.6 ; \mathrm{n}=56)$ respectively. The cumulative mortality was $2.7 \%$ over the entire period of follow-up (7.9 years). The overall Loss to Follow up (LTFU) was 17.7\% $(\mathrm{n}=572)$. There was disproportionately higher LTFU among the controls $20.9 \%(\mathrm{n}=448)$ than among the cases $11.4 \%(\mathrm{n}=$ $124)$, p-value $<0.0001$. Among participants who reported "perfect adherence", only $21.5 \%$ were on zidovudinebased regimens compared with $78.5 \%$ on non-zidovudine based regimens.

Tables 1 and 2 summarize the baseline characteristics of all participants and of cases at switch to second-line ART respectively.

The results for multivariate model are shown in Table 3. We observe that low baseline CD4 count $<50 / \mathrm{ml}$ (H.R $=$ 7.1, 95\% CI $=4.9-10.1$ ); Zidovudine based ART (H.R $1.8,95 \% \mathrm{CI}=1.3-2.5)$ and imperfect ART adherence (H.R $=2.8,95 \% \mathrm{CI}=2.2-3.5)$ were independently associated with treatment failure. All other covariates included did not seem to affect the time to first-line ART failure.

\section{Discussion}

This study was conducted in a large HIV treatment program in western Kenya. We found that low baseline CD4 count $(<50 / \mathrm{ml})$ and poor ART adherence (less than perfect) were independent predictors of first-line treatment failure. This has been established in a number of previous studies [17-19]. However, in this cohort, the baseline WHO clinical stage was not a predictor of treatment failure. It is expected that advanced WHO clinical stage and low CD4 count be in tandem in predicting treatment failure. Patients in WHO stage IV at the time of initiating ART are at a higher risk of treatment failure compared with those in stage I-III [20]. In this setting, we postulate that patients in WHO stage IV are likely to have experienced disproportionately higher and early mortality before first-line treatment failure could be ascertained and this is likely to have masked the true effect of advanced WHO stage on the risk of treatment failure.

In this setting, Zidovudine use as part of the NRTI backbone is associated with treatment failure. Zidovudine is associated with more adverse effects including nausea and vomiting that may potentially reduce treatment adherence [21-23]. Our data showed that majority of patients who reported "perfect adherence" were on non-AZT regimens. Previous clinical trials comparing stavudine-versus zidovudine-based ART have documented better increases from baseline in median CD4 counts in the stavudine arm [24-26]. The clinical implication of this finding is uncertain and more data are required to examine this association conclusively.

We did not find any association between the choice of NNRTI used (Nevirapine or Efavirenz) and treatment failure. Previous studies have suggested that Nevirapine-based ART is marginally less efficacious compared 
A Case-Control Study

Table 1. Comparison of cases and controls.

\begin{tabular}{|c|c|c|c|}
\hline Characteristic & Cases & Controls & p-value \\
\hline Median age at start of ART (IQR) & $36.3(30.6-43.2)$ & $36.5(30.7-43.1)$ & 0.7188 \\
\hline Median time on ART (IQR) & $33.6(\mathrm{IQR}=24-47.4)$ & $48(28.8-62.4)$ & $<0.0001$ \\
\hline \multicolumn{4}{|l|}{ Gender } \\
\hline Male & $415(38.3)$ & $826(38.4)$ & \\
\hline Female & $669(61.7)$ & $1323(61.5)$ & 0.933 \\
\hline \multicolumn{4}{|l|}{ Type of clinic } \\
\hline Urban & $300(27.7)$ & $537(25.0)$ & \\
\hline Rural & $784(72.3)$ & $1612(75.0)$ & 0.0998 \\
\hline \multicolumn{4}{|l|}{ Death } \\
\hline Yes & $33(3.0)$ & $56(2.6)$ & \\
\hline No & $1051(97.0)$ & $2093(97.4)$ & 0.4951 \\
\hline \multicolumn{4}{|l|}{ Lost to follow-up } \\
\hline Yes & $124(11.4)$ & $448(20.9)$ & \\
\hline No & $960(88.6)$ & $1701(79.1)$ & $<0.0001$ \\
\hline \multicolumn{4}{|l|}{ WHO stage } \\
\hline stage 1 & $245(22.6)$ & $717(33.4)$ & \\
\hline stage 2 & $284(26.2)$ & $455(21.2)$ & \\
\hline stage 3 & $488(45.0)$ & $855(39.8)$ & \\
\hline stage 4 & $67(6.2)$ & $122(5.7)$ & $<0.0001$ \\
\hline Median CD4 n (IQR) & $80(32-177)$ & $194(112-324)$ & $<0.0001$ \\
\hline \multicolumn{4}{|l|}{ CD4 category } \\
\hline$<50$ & $376(34.7)$ & $230(10.7)$ & \\
\hline $50-100$ & $240(22.2)$ & $243(11.3)$ & \\
\hline $100-250$ & $312(28.8)$ & $887(41.3)$ & \\
\hline$>250$ & $155(14.3)$ & $789(36.7)$ & $<0.0001$ \\
\hline \multicolumn{4}{|l|}{ Hgb category at baseline } \\
\hline$<10$ & $268(25.0)$ & $482(23.2)$ & \\
\hline$\geq 10$ & $802(75.0)$ & $1597(76.8)$ & 0.5493 \\
\hline \multicolumn{4}{|l|}{ BMI category at baseline } \\
\hline$<18.5$ & $338(31.2)$ & $607(28.2)$ & \\
\hline$\geq 18.5$ & $746(68.8)$ & $1542(71.8)$ & 0.0834 \\
\hline \multicolumn{4}{|l|}{ HIV disclosure } \\
\hline Yes & $726(67.0)$ & $1439(67.0)$ & \\
\hline No & $358(33.0)$ & $710(33.0)$ & 0.9942 \\
\hline \multicolumn{4}{|l|}{ Travel time } \\
\hline$<30$ minutes & $311(28.7)$ & $633(29.5)$ & \\
\hline $30-60$ minutes & $336(31.0)$ & $634(29.5)$ & \\
\hline $1-2 \mathrm{hrs}$ & $276(25.5)$ & $533(24.8)$ & \\
\hline$>2 \mathrm{hrs}$ & $161(14.8)$ & $349(16.2)$ & 0.7295 \\
\hline \multicolumn{4}{|l|}{ ART perfect adherence } \\
\hline Yes & $555(51.2)$ & $1608(74.8)$ & \\
\hline No & $529(48.8)$ & $541(25.2)$ & $<0.0001$ \\
\hline \multicolumn{4}{|l|}{ Discordant at baseline } \\
\hline Yes & $59(5.4)$ & $133(6.2)$ & \\
\hline No & $453(41.8)$ & 937 (43.6) & \\
\hline Unknown & $572(52.8)$ & $1078(50.2)$ & 0.1399 \\
\hline \multicolumn{4}{|l|}{ First-line ART regimens } \\
\hline \multicolumn{4}{|l|}{ Zidovudine vs stavudine } \\
\hline Zidovudine based regimen & $232(22.1)$ & $435(21.1)$ & \\
\hline Stavudine based regimen & $816(77.9)$ & $1629(78.9)$ & 0.4928 \\
\hline \multicolumn{4}{|l|}{ Efavirenz vs nevirapine } \\
\hline Efavirenz based regimen & $155(14.8)$ & $272(13.5)$ & \\
\hline Nevirapine based regimen & $894(85.2)$ & $1739(86.5)$ & 0.3436 \\
\hline
\end{tabular}


A Case-Control Study

Table 2. Characteristics of cases at switch to second line.

\begin{tabular}{ccc}
\hline Median viral load & $\mathrm{n}=1014$ & $60896.5(\mathrm{IQR}=24,091.8-178,583)$ \\
Median CD4 count & $\mathrm{n}=1080$ & $114.6(\mathrm{IQR}=61-182)$ \\
Median Hb & $\mathrm{n}=1058$ & $12.4(\mathrm{IQR}=10.9-13.8)$ \\
Median BMI & $\mathrm{n}=1084$ & $20.9(\mathrm{IQR}=18.7-23.2)$ \\
\hline
\end{tabular}

Table 3. Stratified Cox model of factors associated with first-line ART failure.

\begin{tabular}{|c|c|c|c|c|c|c|}
\hline \multirow{2}{*}{ Variables } & \multicolumn{3}{|c|}{ Unadjusted } & \multicolumn{3}{|c|}{ Adjusted } \\
\hline & p-value & H.R & $95 \%$ CI & p-value & H.R & $95 \%$ CI \\
\hline \multicolumn{7}{|l|}{ Clinic type } \\
\hline Urban & 0.592 & 1.05 & $0.871-1.274$ & 0.1527 & 1.04 & $0.803-1.347$ \\
\hline Rural $^{*}$ & & 1 & & & 1 & \\
\hline \multicolumn{7}{|l|}{ Education level } \\
\hline Secondary/tertiary & 0.844 & 1.02 & $0.852-1.217$ & 0.544 & 1.07 & $0.855-1.345$ \\
\hline Primary $^{*}$ & & 1 & & & 1 & \\
\hline \multicolumn{7}{|l|}{ CD4 category } \\
\hline$<50$ & $<0.0001^{* *}$ & 7.49 & $5.571-10.081$ & $<0.0001^{* *}$ & 7.07 & $4.918-10.149$ \\
\hline $50-100$ & $<0.0001^{* *}$ & 4.5 & $3.343-6.064$ & $<0.0001^{* *}$ & 4.37 & $3.036-6.302$ \\
\hline $100-250$ & $0.0003^{* *}$ & 1.6 & $1.242-2.051$ & $0.004^{* *}$ & 1.57 & $1.160-2.130$ \\
\hline \multicolumn{7}{|l|}{ WHO stage at baseline } \\
\hline High (stage 3 or 4 ) & 0.101 & 1.15 & $0.973-1.354$ & 0.688 & 0.95 & $0.756-1.203$ \\
\hline Low (stage 1 or 2$)^{*}$ & & 1 & & & 1 & \\
\hline \multicolumn{7}{|l|}{ BMI category } \\
\hline$<18.5$ & 0.671 & 1.04 & $0.870-1.242$ & 0.053 & 0.78 & $0.606-1.003$ \\
\hline$\geq 18.5^{*}$ & & 1 & & & 1 & \\
\hline \multicolumn{7}{|l|}{ Hemoglobin category } \\
\hline$<10$ & 0.67 & 1.04 & $0.855-1.276$ & 0.602 & 1.07 & $0.819-1.410$ \\
\hline$\geq 10^{*}$ & & 1 & & & 1 & \\
\hline \multicolumn{7}{|l|}{ ART imperfect adherence } \\
\hline Yes & $<0.0001^{* *}$ & 3.02 & $2.511-3.630$ & $<0.0001^{* *}$ & 2.77 & $2.199-3.494$ \\
\hline \multicolumn{7}{|l|}{ HIV disclosure status } \\
\hline No & 0.5676 & 0.95 & $0.796-1.133$ & 0.393 & 1.11 & $0.872-1.417$ \\
\hline Yes* & & 1 & & & 1 & \\
\hline \multicolumn{7}{|l|}{ Travel time } \\
\hline 2 hours & 0.386 & 0.89 & $0.694-1.152$ & 0.709 & 0.94 & $0.677-1.304$ \\
\hline $1-2$ hours & 0.698 & 0.96 & $0.767-1.194$ & 0.933 & 1.01 & $0.750-1.368$ \\
\hline $30-60$ minutes & 0.542 & 1.07 & $0.866-1.314$ & 0.864 & 1.02 & $0.777-1.351$ \\
\hline$<30$ minutes $^{*}$ & & 1 & & & 1 & \\
\hline \multicolumn{7}{|l|}{ Discordance status } \\
\hline Yes & 0.1833 & 0.99 & $0.688-1.424$ & 0.171 & 1.39 & $0.867-2.235$ \\
\hline Unknown & 0.9558 & 1.12 & $0.946-1.340$ & 0.596 & 1.07 & $0.842-1.348$ \\
\hline $\mathrm{No}^{*}$ & & 1 & & & 1 & \\
\hline \multicolumn{7}{|l|}{ ART regimens } \\
\hline Zidovudine (vs stavudine) & $0.009^{* *}$ & 1.37 & $1.082-1.738$ & $0.001^{* *}$ & 1.76 & $1.254-2.475$ \\
\hline Nevirapine (vs efervirenz) & 0.231 & 0.86 & $0.674-1.000$ & 0.536 & 0.93 & $0.654-1.247$ \\
\hline
\end{tabular}

*Reference category; ${ }^{* *}$ Significant at $(\mathrm{p}<0.05)$. 
with Efavirenz-based ART in terms of virological and immunological outcomes [27-29]. Our study did not demonstrate any differences between the two NNRTI's probably because they are very minor and would need a much larger cohort to fully examine this association.

The overall cumulative mortality in this cohort was low at $2.7 \%$ compared to other similar cohorts which reported cumulative mortality rates of $4 \%-16 \%$ [29-31]. This may in part, be due to the impact of Isoniazid and Co-trimoxazole Prophylaxis Therapy practiced in this setting which would have the effect of limiting TB, pneumonia, toxoplasmosis and other opportunistic infections that are responsible for increased mortality in HIV-1 infected patients [32-35]. Further, AMPATH has established a model care provision known as the High Risk Express Care (HREC) system, in which patients initiating ART with a CD4 cell count $<100 / \mathrm{ml}$ have rapid weekly or bi-weekly contact with nurses. This enables early identification and treatment of opportunistic infections and has been shown to reduce mortality by an estimated $40 \%$ [36]. The rate of LTFU was high at $17.7 \%$ with higher rates in the control arm than amongst the cases. We postulate that the controls were likely to have been healthier which made it easier for them to transfer or drop out of clinic.

Variables that were not significantly associated with risk of treatment failure included clinic type (rural versus urban), travel time to clinic, level of education, baseline hemoglobin levels, baseline BMI and HIV discordance and disclosure status. One study documented that care provided in village clinics was less likely to achieve virological suppression compared to care provided at county level hospital [10]. Our study found no differences between urban versus rural centres probably because similar level of care and ART protocols are employed across the board. There are few studies examining these other factors and data are scanty to make a strong conclusion.

\section{Study Limitations}

Baseline viral load testing is not routinely done in this setting. It would have been important to examine the association between baseline viral load and risk of first-line ART failure. In addition, treatment monitoring is based on clinical and immunological criteria and viral load assay is only done when treatment failure is suspected based on these other parameters. Thus, it is possible that we inadvertently failed to include patients with virological failure who had not met the criteria for immunological nor clinical failure in the cases arm. Furthermore, we were not able to assess the levels and impact of primary ART resistance to treatment failure since this test is not routinely available.

\section{Conclusion}

In this setting, low baseline CD4 count, zidovudine-based ART and imperfect adherence are associated with firstline treatment failure. Earlier initiation of ART, as well as developing new strategies to enhance adherence are key priorities in sustaining the first-line regimen.

\section{Acknowledgements}

We thank the patients, clinicians and the data team who made this work possible.

\section{REFERENCES}

[1] P. Lewthwaite and E. Wilkins, "Natural History of HIV/ AIDS," Medicine, Vol. 37, No. 7, 2009, pp. 333-337. doi:10.1016/j.mpmed.2009.04.015

[2] A. Mindel and M. Tenant-Flowers, "Natural History and Management of Early HIV Infection," BMJ, Vol. 322, No. 7297, 2001, pp. 1290-1293. doi:10.1136/bmj.322.7297.1290

[3] A. Siika, P. Ayuo, J. Sidle, A. Mwangi, K. Wools-Kaloustian, S. Kimaiyo, et al., "Admission Characteristics, Diagnoses and Outcomes of HIV-Infected Patients Registered in an Ambulatory HIV-Care Programme in Western Kenya," East African Medical Journal, Vol. 85, No. 11, 2008, pp. 523-528.

[4] R. S. Hogg, B. Yip, C. Kully, K. Craib, M. O’Shaughnessy, M. Schechter, et al., "Improved Survival among HIV-Infected Patients after Initiation of Triple-Drug Antiretroviral Regimens," Canadian Medical Association Journal, Vol. 160, No. 5, 1999, pp. 659-665.

[5] M. Egger, B. Hirschel, P. Francioli, P. Sudre, M. Wirz, M. Flepp, et al., "Impact of New Antiretroviral Combination Therapies in HIV Infected Patients in Switzerland: Prospective Multicentre Study. Swiss HIV Cohort Study," BMJ, Vol. 315, No. 7117, 1997, pp. 1194-1199. doi:10.1136/bmj.315.7117.1194

[6] O. Keiser, H. Tweya, P. Braitstein, F. Dabis, P. MacPhail, A. Boulle, et al., "Mortality after Failure of Antiretroviral Therapy in Sub-Saharan Africa," Tropical Medicine \& International Health, Vol. 15, No. 2, 2010, pp. 251-258. doi:10.1111/j.1365-3156.2009.02445.x

[7] A. Mocroft, B. Ledergerber, J. P. Viard, S. Staszewski, M. Murphy, A. Chiesi, et al., "Time to Virological Failure of 3 Classes of Antiretrovirals after Initiation of Highly Active Antiretroviral Therapy: Results from the EuroSIDA Study Group," The Journal of Infectious Diseases, Vol. 190, No. 11, 2004, pp. 1947-1956. doi:10.1086/425424

[8] D. Cadosch, S. Bonhoeffer and R. Kouyos, "Assessing the Impact of Adherence to Anti-Retroviral Therapy on Treatment Failure and Resistance Evolution in HIV," Journal of the Royal Society, Interface/the Royal Society, Vol. 9, No. 74, 2012, pp. 2309-2320.

[9] M. I. Datay, A. Boulle, D. Mant and P. Yudkin, "Associations with Virologic Treatment Failure in Adults on Antiretroviral Therapy in South Africa," Journal of Ac- 
quired Immune Deficiency Syndromes, Vol. 54, No. 5, 2010, pp. 489-495. doi:10.1097/QAI.0b013e3181d91788

[10] Y. Ma, D. Zhao, L. Yu, M. Bulterys, M. L. Robinson, Y. Zhao, et al., "Predictors of Virologic Failure in HIV-1Infected Adults Receiving First-Line Antiretroviral Therapy in 8 Provinces in China," Clinical Infectious Diseases, Vol. 50, No. 2, 2010, pp. 264-271. doi:10.1086/649215

[11] P. R. Harrigan, R. S. Hogg, W. W. Y. Dong, B. Yip, B. Wynhoven, J. Woodward, et al., "Predictors of HIV DrugResistance Mutations in a Large Antiretroviral-Naive Cohort Initiating Triple Antiretroviral Therapy," Journal of Infectious Diseases, Vol. 193, No. 3, 2005, pp. 339347. doi:10.1086/427192

[12] Kenya Aids Indicator Survey, 2007. http://www.nacc.or.ke/nacc\%20downloads/official_kais_r eport_2009.pdf

[13] W. M. Tierney, J. K. Rotich, T. J. Hannan, A. Siika, P. G. Biondich, B. W. Mamlin, et al., "The AMPATH Medical Record System: Creating, Implementing, and Sustaining an Electronic Medical Record System to Support HIV/ AIDS Care in Western Kenya," Study in Health Technology and Informatics, Vol. 129, 2007, pp. 372-376.

[14] SAS Macros, 2012.

http://mayoresearch.mayo.edu/mayo/research/biostat/sas macros.cfm

[15] World Health Organization, "Antiretroviral Therapy for HIV Infection in Adults and Adolescents: Recommendations for a Public Health Approach-2006 Revision," 2012.

http://www.who.int/hiv/pub/guidelines/artadultguidelines. pdf

[16] B. Langholz and D. C. Thomas, "Nested Case-Control and Case-Cohort Methods of Sampling from a Cohort: A Critical Comparison," American Journal of Epidemiology, Vol. 131, No. 1, 1990, pp. 169-176.

[17] N. Khienprasit, R. Chaiwarith, T. Sirisanthana and K. Supparatpinyo, "Incidence and Risk Factors of Antiretroviral Treatment Failure in Treatment-Naïve HIV-Infected Patients at Chiang Mai University Hospital, Thailand," AIDS Research and Therapy, Vol. 8, No. 1, 2011, p. 42. doi:10.1186/1742-6405-8-42

[18] A. Soria, K. Porten, J.-C. Fampou-Toundji, L. Galli, R. Mougnutou, V. Buard, et al., "Resistance Profiles after Different Periods of Exposure to a First-Line Antiretroviral Regimen in a Cameroonian Cohort of HIV Type-1Infected Patients," Antiviral Therapy (Lond.), Vol. 14, No. 3, 2009, pp. 339-347.

[19] J.-F. Faucher, B. Challier, C. Chirouze, C. Drobacheff, P. Fischer, J.-M. Lang, et al., "Predictive Factors of Virological Response to Primary Antiretroviral Treatment," La Presse Médicale, Vol. 33, No. 5, 2004, pp. 310-315. doi:10.1016/S0755-4982(04)98570-3

[20] D. T. M. Huong, W. Bannister, P. T. Phong, O. Kirk and L. Peters, "Factors Associated with HIV-1 Virological Failure in an Outpatient Clinic for HIV-Infected People in Haiphong, Vietnam," International Journal of STD \& AIDS, Vol. 22, No. 11, 2011, pp. 659-664.

\section{doi:10.1258/ijsa.2011.010515}

[21] G. I. Eluwa, T. Badru and K. J. Akpoigbe, “Adverse Drug Reactions to Antiretroviral Therapy (ARVs): Incidence, Type and Risk Factors in Nigeria," BMC Clinical Pharmacology, Vol. 12, 2012, p. 7 . doi:10.1186/1472-6904-12-7

[22] E. DeJesus, G. Herrera, E. Teofilo, J. Gerstoft, C. B. Buendia, J. D. Brand, et al., "Abacavir versus Zidovudine Combined with Lamivudine and Efavirenz, for the Treatment of Antiretroviral-Naive HIV-Infected Adults," Clinical Infectious Diseases, Vol. 39, No. 7, 2004, pp. 1038-1046. doi:10.1086/424009

[23] P. L. Anderson and J. E. Rower, "Zidovudine and Lamivudine for HIV Infection," Clinical Medicine Reviews in Therapeutics, Vol. 2, 2010.

[24] K. E. Squires, R. Gulick, P. Tebas, J. Santana, V. Mulanovich, R. Clark, et al., "A Comparison of Stavudine plus Lamivudine versus Zidovudine plus Lamivudine in Combination with Indinavir in Antiretroviral Naive Individuals with HIV Infection: Selection of Thymidine Analog Regimen Therapy (START I)," AIDS, Vol. 14, No. 11, 2000, pp. 1591-1600. doi:10.1097/00002030-200007280-00015

[25] N. A. Foudraine, J. J. de Jong, G. Jan-Weverling, B. H. van Benthem, J. Maas, I. P. Keet, et al., "An Open Randomized Controlled Trial of Zidovudine plus Lamivudine versus Stavudine plus Lamivudine," AIDS, Vol. 12, No. 12, 1998, pp. 1513-1519. doi:10.1097/00002030-199812000-00014

[26] M. W. Kline, R. B. Van Dyke, J. C. Lindsey, M. Gwynne, M. Culnane, R. E. McKinney Jr., et al., "A Randomized Comparative Trial of Stavudine (d4T) versus Zidovudine (ZDV, AZT) in Children with Human Immunodeficiency Virus Infection. AIDS Clinical Trials Group 240 Team," Pediatrics, Vol. 101, No. 2, 1998, pp. 214-220. doi:10.1542/peds.101.2.214

[27] R. Manfredi, L. Calza and F. Chiodo, "Efavirenz versus Nevirapine in Current Clinical Practice: A Prospective, Open-Label Observational Study," Journal of Acquired Immune Deficiency Syndromes, Vol. 35, No. 5, 2004, pp. 492-502. doi:10.1097/00126334-200404150-00007

[28] C. W. Wester, A. M. Thomas, H. Bussmann, S. Moyo, J. M. Makhema, T. Makhema, et al., "Non-Nucleoside Reverse Transcriptase Inhibitor Outcomes among Combination Antiretroviral Therapy-Treated Adults in Botswana," AIDS, Vol. 24, Suppl. 1, 2010, pp. S27-S36. doi:10.1097/01.aids.0000366080.91192.55

[29] H. Li, W. Guo, H. Li, Z. Wang, Y.-J. Liu, Z.-Y. Bao, et al., "Occurrence of Human Immunodeficiency Virus-1 Resistance through a Six-Year Surveillance in Rural Areas of Henan," Chinese Medical Journal, Vol. 91, No. 21, 2011, pp. 1443-1447.

[30] G. Wandeler, O. Keiser, K. Pfeiffer, S. Pestilli, C. Fritz, N. D. Labhardt, et al., "Outcomes of Antiretroviral Treatment Programs in Rural Southern Africa," Journal of Acquired Immune Deficiency Syndromes, Vol. 59, No. 2, 2012, pp. e9-e16. doi:10.1097/QAI.0b013e31823edb6a

[31] O. Keiser, B. H. Chi, T. Gsponer, A. Boulle, C. Orrell, S. 
Phiri, et al., "Outcomes of Antiretroviral Treatment in Programmes with and without Routine Viral Load Monitoring in Southern Africa," AIDS, Vol. 25, No. 14, 2011, pp. 1761-1769. doi:10.1097/QAD.0b013e328349822f

[32] F. P. Sarasin, A. Perrier and T. Rochat, "Isoniazid Preventive Therapy for Pulmonary Tuberculosis Sequelae: Which Patients up to Which Age?" Tubercle and Lung Disease, Vol. 76, No. 5, 1995, pp. 394-400. doi:10.1016/0962-8479(95)90004-7

[33] C. Kabali, C. F. von Reyn, D. R. Brooks, R. Waddell, L. Mtei, M. Bakari, et al., "Completion of Isoniazid Preventive Therapy and Survival in HIV-Infected, TST-Positive Adults in Tanzania," International Journal of Tuberculosis and Lung Disease, Vol. 15, No. 11, 2011, pp. 15151521. doi:10.5588/ijtld.10.0788

[34] M. Khongphatthanayothin, A. Avihingsanon, N. Teeratakulpisarn, N. Phanuphak, R. Buajoom, P. Suwanmala, et al., "Feasibility and Efficacy of Isoniazid Prophylaxis for Latent Tuberculosis in HIV-Infected Clients Patients in Thailand," AIDS Research and Human Retroviruses, Vol. 28, No. 3, 2012, pp. 270-275. doi:10.1089/aid.2011.0041

[35] P.-L. Lim, J. Zhou, R. A. Ditangco, M. G. Law, T. Sirisanthana, N. Kumarasamy, et al., "Failure to Prescribe Pneumocystis Prophylaxis Is Associated with Increased Mortality, even in the cART Era: Results from the Treat Asia HIV Observational Database," Journal of the International AIDS Society, Vol. 15, No. 1, 2012, p. 1. doi:10.1186/1758-2652-15-1

[36] P. Braitstein, A. Siika, J. Hogan, R. Kosgei, E. Sang, J. Sidle, et al., "A Clinician-Nurse Model to Reduce Early Mortality and Increase Clinic Retention among High-Risk HIV-Infected Patients Initiating Combination Antiretroviral Treatment," Journal of International AIDS Society, Vol. 15, 2012, p. 7. doi:10.1186/1758-2652-15-7 